\title{
ANÁLISIS CUALITATIVO DEL MODELO DE GESTIÓN DE CASOS PARA LA ATENCIÓN DE PERSONAS EN SITUACIÓN DE PLURIPATOLOGÍA Y SUS CUIDADORES FAMILIARES
}

\author{
Sandra Milena Hernández-Zambrano, Ruth Alexandra Castiblanco Montañez, Ana Julia \\ Carrillo Algarra y Laura Catherin Garcia Rodriguez
}

\footnotetext{
${ }^{1}$ Facultad de Enfermería, Fundación Universitaria de Ciencias de la Salud. Bogotá, Colombia. smhernandez3@fucsalud.edu.co; racastiblanco@fucsalud.edu.co; ajcarrillo@fucsalud.edu.co; cathegarcia96@gmail.com
}

\begin{abstract}
Resumen. Introducción; A pesar de las evidencias de efectividad del modelo de Gestión de Casos en resultados en salud, no se ha localizado en la literatura científica experiencias de implementación y evaluación de la efectividad del modelo en el Sistema de Salud Colombiano; Objetivo; comprender la experiencia de las Enfermeras frente a la implementación del modelo de gestión de casos, para la atención de personas en situación de pluripatología y sus cuidadores familiares; Métodos; Estudio descriptivo cualitativo. Se realizaron diarios de campo que recogieron las experiencias significativas de las Enfermeras Gestoras de Casos durante la intervención. Se realizó análisis de contenido. Resultados: Participaron 6 enfermeras gestoras de casos, con una edad media de 31.8, con más de 2 años de experiencia en el ámbito clínico o comunitario, todas contaban con formación de posgrados ya sea de especialización o maestría. Las categorías emergentes fueron: 1. Percepción de enfermería sobre el sujeto de cuidado pluripatológico y su entorno social 2. Experiencia de cuidado de enfermería en la gestión de casos: rol, aportes y dificultades. Conclusiones: La percepción sobre el paciente pluripatológico aunque se centra en los aspectos fisiológicos, integra en en la valoración como en los cuidados las dimensiones espiritual, psicológica y social.
\end{abstract}

Palabras clave: Gestión de Casos; Pluripatología; Cuidados; Cuidadores Familiares; Investigación Cualitativa

NURSE'S EXPERIENCES FACING THE IMPLEMENTATION OF THE CASE MANAGEMENT MODEL, FOR THE CARE OF PEOPLE WITH PLURIPATHOLOGY AND THEIR FAMILY CAREGIVERS

Abstract: Introduction: despite the evidence of the Case Management effectiveness in health outcomes, there is no scientific evidence of the implementation and evaluation of the Colombian Health System effectiveness. Objectives: understand nurses' experiences, facing the implementation of the Case Management Model, for the care of people with pluripathology and their family caregivers. Method: qualitative descriptive study. Field diaries were made, that collected the significant experiences of the Case Managers Nurses during the intervention. All data were analyzed. Results: 6 Case Managers Nurses participated, with an average age of 31.8 years, 2 or more years of experience in the clinic or community field, all of them has specialization or master's degree. The emerging categories were: 1. Nurse's perception about the pluripathological care subject and their social environment. 2. Nursing care experience in Case Management: role, input and difficulties. Conclusions: the perception over people with pluripathology, even it is centered in physiological aspects, in the assessment and care, integrates the spiritual, psychological and socia aspects of the subject.

Key words: Case Management; Pluripathology; Care, Family Caregivers; Qualitative Investigation.

\section{INTRODUCCIÓN}

La transición epidemiológica y demográfica mundial, explica el aumento de las Enfermedades no Transmisibles (ENT), que está asociado a la pérdida de años de vida 
productivos de la población afectada y a la carga familiar, social y económica relacionada con la cronicidad. En el mundo las ENT matan a 41 millones de personas, lo que equivale al $71 \%$ de las muertes que se producen en el mundo, las enfermedades cardiovasculares constituyen la mayoría de las muertes por ENT, seguidas del cáncer, las enfermedades respiratorias y la diabetes (Organización Mundial de la Salud, 2018).

En Colombia más de 110 mil fallecen por enfermedades crónicas como las afecciones cardiovasculares, el cáncer, la diabetes y los padecimientos pulmonares (Organización Panamericana de la Salud, 2018). Esta situación se complejiza porque aumenta la posibilidad que coexista más de una ENT, especialmente en personas de edad avanzada, lo que implica riesgo de causar discapacidades y fragilidad clínica, aumento de la mortalidad y mayor utilización de los servicios de salud: visitas a urgencias, hospitalizaciones evitables, polifarmacia (Ollero Bernabeu et al., 2010; Montes Santiago et al., 2012). Desde la perspectiva de las personas con múltiples enfermedades crónicas, la progresión de las enfermedades tiene un efecto sobre su calidad de vida y deterioro funcional, impactando en su vida diaria e interrumpiendo las actividades familiares; con el avance de la enfermedad, aumentan los reingresos hospitalarios y los periodos críticos por las complicaciones (Morales-Asencio et al., 2016).

Este fenómeno que se conoce como multimorbilidad o pluripatología, conlleva a la emergencia de modelos conceptuales como Kaiser Permamente que propone la estratificación de la persona en situación de cronicidad en función del riesgo, combinando prevención, apoyo a la autogestión, gestión de la enfermedad y gestión de casos en pacientes de alta complejidad (Fernández Moyano et al., 2017). La Gestión de casos (GC) es un rol de Práctica Avanzada en Enfermería, orientado a la provisión de servicios de salud dirigida a pacientes crónicos complejos y cuidadores principales, para dar respuesta a sus necesidades y minimizar la fragmentación de la atención (Hernández-Zambrano et al., 2019). La Sociedad Americana de Gestión de Casos la describe como un "proceso de colaboración mediante el que se valoran, planifican, aplican, coordinan, monitorizan y evalúan las opciones y servicios necesarios para satisfacer las necesidades de salud de una persona, articulando la comunicación y recursos disponibles para promover resultados de calidad y coste-efectivos" (Case Management Society of America, 2002, p. 8).

A pesar de las evidencias de efectividad del modelo de GC en resultados en salud, como sobrecarga del cuidador, utilización de los servicios de salud, coordinación asistencia, 
polifarmacia, calidad de vida, satisfacción con los servicios y contención de costos en salud (Morales-Asencio, 2014), no se ha localizado en la literatura científica experiencias de implementación y evaluación de la efectividad del modelo en el Sistema de Salud Colombiano. En este sentido, se desarrolló un análisis cualitativo para la evaluación del modelo con población crónica compleja. En el proceso de adaptación cultural de la GC, se determinó que el modelo está orientado a la continuidad asistencial, a través de la coordinación entre niveles asistenciales, profesionales y/ o sectores; a la movilización de servicios socio-sanitarios; optimización en la utilización de los servicios; garantizar la calidad de las transiciones y un plan terapéutico individualizado; además de promover el empoderamiento y autonomía en la gestión del plan asistencial del paciente y su cuidador principal (Hernández-Zambrano et al., 2019). Dada la importancia de recoger evidencias cualitativas que apoyen en el modelaje de esta intervención compleja de salud, el objetivo del estudio, es comprender la experiencia de las Enfermeras frente a la implementación del modelo de gestión de casos, para la atención de personas en situación de pluripatología y sus cuidadores familiares.

\section{METODOLOGÍA}

\subsection{Diseño de investigación}

Se llevó a cabo un estudio descriptivo cualitativo, para comprender la experiencia de las enfermeras frente a la implementación del modelo de gestión de casos, para la atención de personas en situación de pluripatología y sus cuidadores familiares. En el campo de la salud comunitaria Swanson destaca la importancia del enfoque cualitativo en la investigación evaluativa, que proporciona evidencias sobre los mecanismos en los que se basan las intervenciones, examina el proceso de implementación de un programa de atención en salud y no solo mide los resultados. (Amezcua \& Hernández Zambrano, 2012; De la Cuesta, 2005)

\subsection{Lugar de estudio}

El estudio se realizó en el ámbito domiciliario de personas en situación de pluripatología, localizado en Bogotá, Colombia.

\subsection{Población de estudio}

Participaron 6 enfermeras que recibieron entrenamiento en el modelo de Gestión de Casos. La intervención estuvo dirigida a 317 pacientes en situación de pluripatología y sus 
cuidadores familiares (Diadas). Finalizaron 272 pacientes, 27 fallecieron y 18 se retiraron del estudio.

\subsection{Criterios de inclusión}

1. Enfermera/o con registro profesional correspondiente para Colombia

2. Experiencia mínima de 2 años en entorno hospitalario o comunitario

3. Formación posgradual de especialización o maestría con competencias clínicas o comunitarias.

4. Entrenamiento en abordaje del paciente crónico complejo y sus cuidadores familiares

Muestreo intencional por conveniencia que cumpliera cons todos los criterios de inclusión.

\subsection{Recolección de datos}

Se realizaron diarios de campo que recogieron las experiencias significativas de las Enfermeras Gestoras de Casos durante la intervención. El proceso de Gestión de Casos se desarrolló durante 12 meses a través de cuatro componentes: 1. La identificación del caso; 2. La valoración personalizada que incluye, la revisión de la complejidad y la evaluación integral exhaustiva, multidimensional de las necesidades de atención sanitaria y social. 3. El diseño, ejecución y evaluación del plan de gestión de casos: plan de cuidado personalizado utilizando taxonomía NANDA, NIC, NOC y plan de coordinación de las intervenciones con otros proveedores y gestión de barreras administrativas y 4 . El cierre del caso. Se realizaron mínimo 6 visitas según la complejidad del paciente (1 visita de valoración integral exhaustiva y multidimensional, 2 visitas de seguimiento domiciliario, 2 seguimientos telefónicos, 1 visita de evaluación) .

Las variables sociodemográficas fueron recolectadas a través de un formato codificado dentro del mismo formato del diario de campo.

\subsection{Análisis de datos}

Se realizó análisis de contenido siguiendo la propuesta de Taylor y Bogdan, adaptada por Amezcua y Gálvez (2002). Se realizó un esquema en espiral que obliga a retroceder una y otra vez a los datos para dar consistencia a las interpretaciones, las etapas no suceden 
unas tras otras como ocurre en el esquema secuencial, las etapas son: preparación de los datos que está orientada a facilitar la mirada reflexiva de investigador a través de presentaciones concentradas como resúmenes estructurados o diagramas; descubrimiento de temas donde se realiza la búsqueda de temas examinando los datos de todos los modos posibles; codificación e interpretación de datos que consiste en la reunión y análisis de todos los datos que refieren a temas, ideas, conceptos, interpretaciones y proposiciones; relativización de los datos cuyo propósito es la interpretación en el contexto en el que fueron recogidos los datos; y garantía del rigor a través de la triangulación entre investigadores.

\subsection{Consideraciones éticas}

El presente estudio de acuerdo con el artículo 11 de la resolución 8430 de 1993 de Colombia, se consideró de mínimo riesgo para la vida y la integridad de los sujetos de investigación. Este proyecto fue aprobado por el comité de ética en investigación en seres humanos CEISH, el día 26 de Mayo de 2017 en Bogotá. Se firmó consentimiento informado y la participación fue voluntaria tanto para los participantes como las enfermeras gestoras de casos.

\section{RESULTADOS Y DISCUSIÓN}

\subsection{Características sociodemográficas}

Participaron seis enfermeras con una edad promedio de 31.8 años, todas tenían más de 2 años experiencia en el ámbito clínico o comunitario y formación de posgrados así: especialización en Enfermería nefrológica del adulto, especialización en epidemiología, especialización en gerencia de instituciones sociales de salud y maestría en salud pública o en enfermería.

\subsection{Categorías emergentes}

- Categoría 1. Percepción de enfermería sobre el sujeto de cuidado pluripatológico y su entorno social

\section{Percepción sobre el sujeto de cuidado}

La condición de pluripatología afecta a los sujetos de cuidado desde lo fisiológico, demandando tratamientos y estancias hospitalarias en algunos casos prolongadas, que generan implicaciones a nivel laboral como pérdida del trabajo, a nivel social, con pérdida de 
relaciones de amigos y familiares, y a nivel emocional, ocasionando sentimientos de aislamiento y deterioro del autoconcepto y autoconfianza derivados de las dinámicas de la enfermedad.

"Ella perdió su trabajo desde que diagnosticaron la primera enfermedad... con el paso del tiempo se presentaron más enfermedades que fueron afectando su estado emocional hasta llegar a un trastorno depresivo recurrente... La paciente ha perdido sus amigos, nadie la volvió a llamar y Ella también reconoce que se ha aislado de las redes sociales, refiere que no le interesa compartir con otras personas y que tampoco tiene mucho tiempo, pues además de su rol como paciente con pluripatología es a la vez cuidadora de sus padres y abuela, quienes son también pacientes crónicos" Participante 2.

Se resalta que, en cuanto a la dimensión espiritual, se fortalecen las creencias en un ser supremo con los procesos de enfermedad, que se traduce en mayor empoderamiento para anteponerse a las dificultades diarias, capacidad para disfrutar cada instante de sus vidas y mayor comprensión sobre la muerte, al no querer representar una carga a sus familiares.

"Pero todos tienen en común qué le piden a Dios que los deje hasta cuando ellos no dependan totalmente de sus familiares, hasta que sean útiles" Participante 1.

A pesar de lo anterior, la mayoría de enfermeras gestoras describen al paciente priorizando los aspectos clínicos sobre los relacionados con las otras dimensiones abordadas que permitan un enfoque integral de cuidado.

Diferentes autores han descrito la espiritualidad como la integración de cuerpo, mente y espíritu, que se traduce en encontrar la paz, armonía y el significado de la vida. Esta Espiritualidad en personas con enfermedades crónicas mejora la respuesta inmunológica, la adaptación frente a la enfermedad, la resolución de problemas y la toma de decisiones en cuanto a la condición de salud o enfermedad. De igual manera permite el manejo de los sentimientos desesperación, depresión, soledad y ansiedad asociados a la cronicidad. (Drutchas y Anandarajah, 2014; Vargas-Escobar, 2017)

\subsection{Percepción de la enfermera sobre el entorno de cuidado y social del paciente}

En procesos de enfermedad o consecuencias que generan cambios en la calidad de vida de las personas, es importante el apoyo familiar, que garantice acompañamiento y fomente la capacidad de afrontamiento y manejo de su condición. 
"Frente a este caso me siento vulnerable, considero que todos estamos expuestos a una situación similar y que enfrentarse a este cambio de vida requiere apoyo de la familia, confianza en sí mismo y sobre todo motivaciones para seguir adelante" Participante 3.

En este sentido, cabe resaltar que este apoyo está presente independientemente de las condiciones socioeconómicas o la presencia de hijos, a través de un cuidador que brinda bienestar. Sin embargo, cuando la condición de pluripatología se presenta en edades tempranas, los sujetos deben desempeñar un doble rol: paciente y cuidador de familiares a cargo.

Estos resultados son similares a lo que menciona Merodio, Acuña y Martínez (2015) en su estudio de la percepción del apoyo familiar, la familia cumple un papel importante en el apoyo instrumental de las personas mayores, el cónyuge y los hijos son los principales proveedores de ayuda económica y de cuidados, pues se requiere garantizar acompañamiento continuo y toma de decisiones de la condición de salud de su familiar enfermo.

Asimismo, las dinámicas del Sistema de Salud promueven la delegación del cuidado directo a la familia del paciente, sin ningún tipo de preparación o en entrenamiento previo, lo que ocasiona que exista desconocimiento sobre el manejo en el proceso de la enfermedad que les permita a los cuidadores dar respuesta a las necesidades de los pacientes; aunque se manifieste interés y motivación por brindar un cuidado integral; actuando como fuente de apoyo principal y asumiendo actividades que suplen desde necesidades básicas, físicas, emocionales y sociales, a otras más complejas como la gestión administrativa de su proceso de atención.

"los cuidadores se dedican a brindar la atención en todas las actividades de rutina, desde la higiene diaria, alimentación, administración de medicamentos, hasta los difíciles trámites para obtener las citas médicas de control" Participante 2.

No obstante, al delegarse todas estas actividades, el familiar que asume el rol de cuidador entra en riesgo de sobrecarga con el transcurso del tiempo por el acompañamiento continuo que demanda la condición de pluripatología.

"El cuidador se siente con muchas responsabilidades con su familiar, el manejo de un paciente con enfermedad mental es "desgastante", cada actividad debe ser realizada en compañía y bajo la supervisión de un cuidador" Participante 2. 
En la literatura también se evidencia que quien asume el rol de cuidador, en varias ocasiones es una mujer del contexto familiar, que con el transcurso del tiempo y debido a la carga de la condición de múltiples condiciones crónicas y la dependencia de la persona al cuidador, se traduce como perjudicial para la salud de las personas cuidadoras (Valverde, 2014).

- Categoría 2. Experiencia del cuidado de enfermería en la gestión de casos: rol, aportes y dificultades

\section{Rol de enfermería en la gestión de casos}

El rol de enfermería en la gestión de casos está definido como la capacidad de brindar cuidados integrales desde el acompañamiento directo en las viviendas y seguimiento telefónico encaminados a fomentar bienestar físico, mental, emocional y social. Para esto, es necesario que los profesionales cuenten con conocimientos especializados tanto clínicos como de procesos administrativos que les permita orientar, educar y/o resolver las inquietudes de sujetos de cuidado y cuidadores que se puedan presentar en el transcurso del proceso de la enfermedad.

"considero que mi visita o llamada podrá ser una oportunidad para brindar un cuidado y orientación que como enfermera compartiré con pacientes y cuidadores"..."En las primeras visitas me he sentido identificada con este Rol como Enfermera; el servicio y la calidad humana son condiciones relevantes para desempeñar la orientación, educación y resolución de inquietudes de los pacientes y cuidadores. Algunas visitas me hacen sonreír porque sé que logró un buen resultado, otras me contagiaron un poco de incertidumbre por las necesidades que vislumbro en algunos hogares" Participante 2.

En pacientes pluripatológicos, dadas sus condiciones clínicas y comorbilidades, las enfermeras gestoras de casos se enfrentaron a condiciones de fin de vida en las que manifestaron acompañamiento dirigido, fomentando la expresión de emociones en los familiares frente a la pérdida y su tránsito por las diferentes etapas del duelo. Además, las enfermeras han aprovechado estos espacios para reconocer a las familias la labor tan importante que realizaron como cuidadores en el proceso de enfermedad de su familiar.

"permitiéndole a la familia primeramente expresar mediante el diálogo sus sentimientos, los dos familiares manifestaron sentir dolor por la pérdida de su ser querido pero también se observa aceptación frente a la situación que ellos estaban viviendo" (...) "es poder reconocer a las familias la labor tan bonita que realizan al poder cuidar, acompañar, brindar una voz de aliento al final de los días de las personas que amamos, ya que ser cuidador no es fácil y más aún cuando quisiéramos hacer más por las personas que amamos y tal vez por desconocimiento sentimos impotencia de no poder hacer más por las personas que amamos" Participante 5. 
Por último, la competencia de comunicación de la enfermera en la gestión de casos de pacientes con pluripatología y su cuidador, es descrito por las profesionales como un pilar importante, puesto que es la principal herramienta para identificar necesidades de salud, brindar cuidados integrales apoyados en otras áreas del sistema y para que los pacientes adquieran compromisos de mejoramiento de su estado de salud, a través de su empoderamiento para la toma de decisiones.

"Considero que fue una visita agradable en la cual se pudo establecer una buena comunicación con la paciente ya que hay un compromiso de por medio donde se enriquece la relación enfermera paciente y seguramente se obtendrá buenos resultados, los cuales serán evaluados en siguiente visita domiciliaria" Participante 4.

Los resultados de este estudio coinciden con Joo y Huber (2018), en su estudio la gestión de casos, el modelo proporciona atención de calidad, continua y centrada en el paciente. Las intervenciones de las enfermeras en este modelo están centradas en evaluar las necesidades de los pacientes y ofrecer planes de atención basados en estas necesidades.

\subsection{Aportes y dificultades de la gestión de casos desde la experiencia de cuidado}

Participar en el modelo de gestión de casos desde la percepción de las enfermeras, ha significado un gran beneficio en los sujetos de cuidado y cuidadores, gracias al apoyo, acompañamiento y cuidados en el proceso de enfermedad.

Asimismo, para las profesionales significa una gran oportunidad para aprender sobre la gestión de casos como un modelo importante y necesario para la población en el contexto Colombiano, debido a las diferentes barreras de atención en salud a las que se enfrentan sobre todo, de tipo administrativas como inconvenientes que se presentan con las EPS en la prestación de los servicios de salud, que en ocasiones dificultan el adecuado seguimiento por parte del equipo de salud y se traduce en deterioro de la condición del paciente 0 reingresos hospitalarios, y al desconocimiento general de la condición en el paciente y su familiar.

\footnotetext{
"se requiere hacer gestión de casos porque existe un gran desconocimiento sobre los cuidados básicos que puede tener el paciente y cuidador en el hogar, los signos y síntomas de la enfermedad, las alternativas de atención con las que cuenta en su EPS y en los lugares cercanos a la Localidad en donde vive, los cuales aportarían interacción con otras personas y porque no experiencias tal vez con personas que tengan su misma enfermedad"... "La "Gestión de Casos" en Colombia debe verse como
} 
una prioridad, el contacto con el ser humano es cuidado personalizado, es para ellos "sentir que alguien está pendiente", que sonríe con ellos y los escucha" Participante 2.

Las enfermeras de casos de este estudio coinciden con lo descrito por Wynia, et. al (2018) en cuanto a que sus enfermeras experimentaron que brindar la atención integral y centrada en la persona era muy gratificante, pero requería de un cambio importante en el rol que desempeñan. El proceso les brindó oportunidades para aprendizaje y crecimiento personal y profesional.

En consecuencia, estas barreras han repercutido de manera negativa en la población que ha sido expuesta a diferentes circunstancias en su proceso de enfermedad que los lleva a rechazar el acercamiento o acompañamiento por parte de la enfermera y en algunos casos estas han sido víctimas de agresiones verbales y de reclamaciones de incumplimiento en su proceso de atención, imposibilitando que se pueda cumplir con el objetivo del modelo.

"Encuentro que si los pacientes tuvieran un seguimiento adecuado por parte de la EPS, los ingresos y estancias hospitalarias podrían disminuir sustancialmente porque se podrían prevenir las complicaciones de cada patología al identificar los signos de alerta de las mismas" Participante 2.

"he tenido momentos donde nuestros pacientes están tan cargados de esa parte negativa que les genera las barreras a las cuales los someten las EPS, que cuando se realiza alguno de los seguimientos ellos se desquitan con nosotros a veces hasta el punto de agresiones verbales que pueden llegar a herir... es triste no poder contribuir a la mejora de la calidad de vida de ese paciente" Participante 1.

Las enfermeras gestoras de casos tuvieron dificultad en el proceso de la implementación, dado las condiciones geográficas de la ciudad de Bogotá, pues algunos participantes se encontraban en lugares muy apartados, de difícil acceso o con actitudes agresivas que impiden las relaciones terapéuticas.

\section{CONCLUSIONES}

La percepción de la enfermera sobre el paciente pluripatológico aunque se centra en la descripción de los aspectos fisiológicos y clínicos del paciente, integra tanto en la valoración como en los cuidados las dimensiones espiritual, psicológica y social, y las cataloga como indispensables para cumplir las metas de la gestión del caso.

Se evidencia delegación del cuidado y responsabilidades administrativas de la atención a los familiares, ante lo cual se recomienda brindar apoyo, valoración y formación a los 
cuidadores desde la implementación del modelo de gestión de casos para evitar la sobrecarga y deterioro en su calidad de vida.

En el complejo escenario de la atención en salud y el cuidado de las personas a través de un Modelo de Gestión de casos, la investigación cualitativa permite conocer las diferentes verdades, lo que se traduce en conocer diferentes puntos de vista sobre la implementación del modelo en el contexto Colombiano, desde los ojos de las experiencias recolectadas por las enfermeras que participaron en este proceso, y ofrece las bases teóricas para acciones concretas en un futuro.

Se recomienda, abordar el efecto de la gestión de casos en la disminución de barreras de atención en salud que impiden el acceso oportuno para reducir complicaciones de su condición crónica, reingresos hospitalarios y sobrecostos de atención.

Agradecimientos: El producto presentado se realizó gracias a la enfermeras Gestoras de Casos, que proporcionaron la información de análisis en sus diarios de campo

Financiación: Departamento Administrativo de Ciencia, Tecnología e Innovación de Colombia (Colciencias), a través de la convocatoria 777 para proyectos de Ciencia, Tecnología e Innovación en Salud — 2017, número de contrato 848-2017.

\section{REFERENCIAS}

Amezcua, M. \& Hernández Zambrano, S. M. (2012). Investigación sobre el cotidiano del sujeto: oportunidades para una ciencia aplicada. Texto Contexto Enferm, 21(3), 675-683.

Amezcua, M., \& Gálvez Toro, A. (2002). Los modos de análisis en Investigación Cualitativa en Salud: perspectiva crítica y reflexiones en voz alta. Rev Esp Salud Pública, 76(5), 423-436.

Case Management Society of America. Standards of Practice for Case Management; 2002.

De la Cuesta C. (2005). La contribución de la evidencia cualitativa al campo del cuidado y la salud comunitaria. Index Enfermería, 14(50), 47-52.

Drutchas, A. Anandarajah, G. (2014). Spirituality and Coping with Chronic Disease in Pediatrics. R I Med J; 97(3): 26-30.

Fernández Moyano, A., Machín Lázaro, J. M., Martín Escalante, M. D., Aller Hernandez, M. B., \& Vallejo Maroto, I. (2017). Modelos de atención al paciente pluripatológico. Rev Clin Esp, 217(6), 351-358.

Hernández-Zambrano, S. M., Mesa-Melgarejo, L., Carrillo-Algarra, A. L., Castiblanco-Montañez, R. A., Chaparro-Diaz, L., \& Carreño-Moreno, S. P., et al. (2019). Effectiveness of a case management model for the comprehensive provision of health services to multi-pathological people. J Adv Nurs, 75(3), 665-675. doi: 10.1111/jan.13892.

Hernández Zambrano, S. M., Carrillo Algarra, A. J., Castiblanco Montañez, R. A., Chaparro Díaz, O. L., Carreño Moreno, S. P., \& Gonzalo Jiménez, E. (2019). Componentes del modelo de gestión de casos en la atención de la persona en condición de pluripatología y sus cuidadores familiares en Colombia. Paraninfo Digital, 13(29), 1-5. 
Joo, JY; Huber, DL. (2018) Scoping Review of Nursing Case Management in the United States. Clin Nurs Res, 27(8):1002-1006. DOI: 10.1177/1054773817717861.

Merodio Pérez, Zoyla; Rivas Acuña, Valentina; Martínez Serrano, Amalia. (2015). Percepción del apoyo familiar y dificultades relacionadas con la diabetes en el adulto mayor. Horizonte Sanitario, 14 (1), 14-20.

Montes Santiago, J., Casariego Vales, E,, Toro Santos, M., \& Mosquera, E (2012). La asistencia a pacientes crónicos y pluripatológicos. Magnitud e iniciativas para su manejo: La Declaración de Sevilla. Situación y propuestas en Galicia. J Galicia Clin 2012, 73 (Supl.1), S7-S14.

Morales-Asencio, J. M., Martin-Santo,S F. J., Kaknani, S., Morilla-Herrera, J. C., Cuevas Fernández-Gallego, M., \& García-Mayor S, et al. (2016) Living with chronicity and complexity: Lessons for redesigning case management from patients' life stories - A qualitative study. J Eval Clin Pract, 22(1), 122-32.

Morales-Asencio, J. M. (2014). Gestión de casos y cronicidad compleja: conceptos, modelos, evidencias e incertidumbres. Enferm Clin, 24(1), 23-34.

Ollero Bernabeu M., Rincón M., Upshur R., Berstein B. [Colaboradores principales]. (2010) The language of polypathology. En: Jadad AR, Cabrera A, Martos F, Smith R, Lyons RF. When people live with multiple chronic diseases: a collaborative approach to anemerging global challenge. Granada: Escuela Andaluza de Salud Pública. Disponible en: http://www.opimec.org/equipos/when-people-live-with-multiple-chronicdiseases

Organización Mundial de la Salud (2018). Enfermedades no transmisibles. Disponible en: https://www.who.int/es/news-room/fact-sheets/detail/noncommunicable-diseases

Organización Panamericana de la Salud (2018). Las enfermedades no transmisibles (ENT), nuestro reto. Disponible en: https://www.paho.org/col/index.php?option=com_content\&view=article\&id=1756:lasenfermedades-no-transmisibles-ent-nuestro-reto\&Itemid $=487$

Valverde Jiménez, María; López Benavente, Y; Hernández Corominas, María de los ángeles; Gómez Ruiz; M; Blázquez Pedrero, M; Echevarría Pérez P. (2014). Enfermera Gestora de Casos del Servicio Murciano de Salud: Un año de puesta en marcha del programa. Enferm Glob, 13(4), 57-69.

Vargas-Escobar, Lina María (2017). Intervención educativa para enfermería: cuidado espiritual durante la enfermedad crónica. Aquichan, 17(1),30-41.

Wynia, K; Uittenbroek, RJ; Van der Mei, SF; Slotman, K; Reijneveld, SA. (2018) Experiences of case managers in providing person-centered and integrated care based on the Chronic Care Model: A qualitative study on embrace. PLoS One, 13(11):1-16. 